\title{
End Relative To Reference Code
}

National Cancer Institute

\section{Source}

National Cancer Institute. End Relative To Reference Code. NCI Thesaurus. Code C93561.

A coded value specifying when an event ended with respect to the sponsor-defined reference period. 